\title{
Review: antidepressants are moderately effective in people with depression and a substance dependence disorder
}

Nunes EV, Levin FR. Treatment of depression in patients with alcohol or other drug dependence. A meta-analysis. JAMA 2004;291:1887-96.

What is the effect of antidepressants in people with combined depression and substance dependence disorders?

METHODS

$-$

Design: Systematic review with meta-analysis

4

Data sources: PubMed, MEDLINE, and Cochrane database searched from 1970 to December 2003 plus hand search of reference lists and contact with 11 experts.

Study selection and analysis: Inclusion criteria: prospective, double blind, randomised controlled trials in people diagnosed (according to standard criteria) with a current alcohol or substance dependence (opiate, cocaine, stimulant, or sedativehypnotic substances) and a unipolar depressive disorder (including major depression or dysthymia). Analysis: pooled effect sizes were assessed.

国业事

Outcomes: Change in depressive symptoms (Hamilton Depression Scale, where 10-20 indicates mild to moderate depression and $>20$ indicates severe depression).

\section{MAIN RESULTS}

Fourteen RCTs $(\mathrm{n}=848)$ met inclusion criteria: alcohol dependence, eight RCTs; methadone maintained opiate dependence, four RCTS; cocaine dependence, two RCTs. Antidepressants produced a clinically significant response in 52\% compared with 38\% placebo (percentage difference $16.8 \%, 95 \%$ CI $6.9 \%$ to $26.7 \%$ ). Antidepressants produced a pooled effect size for the Hamilton Depression Scale of 0.38 (95\% CI, 0.18 to 0.58 )

\section{CONCLUSIONS}

Antidepressant treatment does benefit people with both depression and substance dependence disorders. However, therapy, which specifically addresses addiction, is also recommended.

\section{NOTES}

Authors found significant heterogeneity in treatment effects in the studies, so results should be treated with caution. They note that

For correspondence: Edward Nunes, New York State Psychiatric Institute, Riverside Dr, New York, USA; nunesed@pi.cpmc.columbia.edu

Sources of funding: the National Institute on Drug Abuse and the New York State Psychiatric Institute. the initial diagnosis of depression is critical for differentiating the symptoms of depression from substance related depression effects.

\section{Commentary}

7 he most innovative aspect of this investigation is the inclusion of people with both alcohol and drug dependence. Previous studies have tended to focus on one or the other comorbidity (that is, alcohol dependence and depression or drug dependence and depression). The study raises the importance of cross training-that is, people primarily specialised in addictions gaining expertise in psychiatric disorders, and professionals specialising in psychiatry and psychology gaining expertise in addiction medicine.

Psychiatry and addiction medicine often involve extensive patient planning conferences with interdisciplinary staff, during which the issue of differential diagnosis in the context of treatment planning repeatedly arises. In the first few days, the sequelae of clinical symptoms associated with detoxification hamper the ability to rule out depression and other Axis I disorders, although medical records may confirm previous major depressive episodes. This study supports the importance of waiting for at least a week of abstinence before establishing a diagnosis of depression. Clinicians with specialised training in addiction, and experience of treating dually (and multiply) diagnosed people are familiar with the standard clinical practice of first conducting psychological interventions before beginning pharmacological treatment. If symptoms persist after several sessions of cognitive behavioural therapy or a variant of this approach, then medication can be considered. It is reassuring to see that the findings from this work support a model of psychological treatment that requires the provider to use a sequential model of care that matches the clinical presentation of the individual.

If antidepressant medications are indicated for dually diagnosed patients, the findings that they can be effective (when used at adequate doses for at least six weeks) in people accurately diagnosed with major depression or dysthymia are clinically relevant. However, the fact that depression is successfully treated does not necessarily mean that associated alcohol or substance dependence will improve as well. The study reinforces the importance of implementing a separate addiction treatment protocol with such patients. In the case of alcohol dependence, naltrexone can be administered along with psychosocial interventions designed to target addiction (for example, 12 step programmes, motivational interviewing, cognitive behavioural therapy relapse prevention, contingency management, and so on). With comorbid opiate dependence, buprenorphine-along with psychosocial interventions targeting the addiction - may be indicated as well.

Charlene E Le Fauve, PhD Division of State and Community Assistance (DCSA), Rockville, MD, USA 